Pacific

Journal of

Mathematics

THE CORRESPONDENCE BETWEEN AUGMENTATIONS AND RULINGS FOR LEGENDRIAN KNOTS

LENHARD L. NG AND JoshuA M. SABLofF 


\title{
THE CORRESPONDENCE BETWEEN AUGMENTATIONS AND RULINGS FOR LEGENDRIAN KNOTS
}

\author{
LENHARD L. NG AND JoshuA M. SABLOFF
}

\begin{abstract}
We strengthen the link between holomorphic and generating-function invariants of Legendrian knots by establishing a formula relating the number of augmentations of a knot's contact homology to the complete ruling invariant of Chekanov and Pushkar.
\end{abstract}

\section{Introduction}

The theory of Legendrian knots in the standard contact $\mathbb{R}^{3}$ up to Legendrian isotopy is a subject of much recent interest. Beginning in the late 1990's, two nonclassical invariants of such Legendrian knots emerged. The first is based on the contact homology theory of Eliashberg and Hofer [Eliashberg 1998], and was couched in combinatorial form by Chekanov [2002a]. This invariant is a differential graded algebra that counts certain holomorphic disks in the symplectization of $\mathbb{R}^{3}$. The second is the ruling invariant of Chekanov and Pushkar [2005], which is derived from the theory of generating functions but has a simple combinatorial definition. See [Chekanov 2002b] for a brief introduction to both invariants.

It seems likely that the information contained in the Chekanov-Pushkar ruling invariant is in some way the same as that contained in the linear level of the contact homology DGA. The first link between the two theories was provided by Fuchs [2003], who introduced the concept of a ruling independently of ChekanovPushkar and demonstrated that the existence of a ruling implies the existence of a so-called augmentation of the contact homology DGA. Fuchs and Ishkhanov [2004] and, independently, the second author [Sabloff 2005] proved the converse. In fact, a more general sort of correspondence seems to hold between generatingfunction invariants and linearized contact homology; see [ $\mathrm{Ng}$ and Traynor 2004] for evidence in $J^{1}\left(S^{1}\right)$.

Here we deepen the link between contact homology and rulings by showing that there is a many-to-one correspondence between augmentations and rulings for a plat-position front of a Legendrian knot. (This is in fact true for any Legendrian

MSC2000: primary 57R17; secondary 53D40, 57M25.

Keywords: Legendrian knots, generating functions, contact homology, augmentations.

$\mathrm{Ng}$ is supported by the American Institute of Mathematics. 
front, but we restrict to plat position for ease of exposition.) An algorithm was given in [Sabloff 2005] for obtaining a ruling of a plat-position front from an augmentation of its DGA. We show that the number of augmentations that yield a particular ruling via the algorithm is determined by the combinatorics of the ruling (Theorem 2). In particular, the total number of augmentations of the DGA of any Legendrian knot can be calculated from its ruling invariant (Corollary 3 ).

\section{Main results}

We begin by introducing the terminology necessary to state our main results. See, for example, [Sabloff 2005] for the precise definitions and conventions which we will use: contact homology DGA, stable tame isomorphism, and algebraic stabilization. As some geometric conventions and the augmentation and ruling constructions are central to this paper, we will review them in more detail.

Let $K$ be a Legendrian knot. A front diagram of $K$ in the standard contact space $\left(\mathbb{R}^{3}, d z-y d x\right)$ is simply the image of the projection of $K$ to the $x z$ plane. A front diagram is in plat position if all of the left cusps have the same $x$ coordinate, all of the right cusps have the same $x$ coordinate, and no two crossings have the same $x$ coordinate. For example, the front diagram of the Legendrian trefoil in Figure 1 is in plat position.

Next, we describe some algebraic constructs related to the contact homology DGA. Let $K$ be a Legendrian knot in the standard contact $\mathbb{R}^{3}$. Its contact homology is the homology of a semifree unital differential graded algebra $(\mathscr{A}, \partial)$ with coefficients in $\mathbb{Z} / 2$ and grading over $\mathbb{Z} / 2 r(K)$. Here $r(K)$ is the rotation number of $K$ and $\mathscr{A}$ is generated by the crossings and right cusps of a front diagram $D$ of $K$. Of particular interest for this paper are the gradings of the crossings: define a locally constant function $m$ from the complement of the cusps in $K$ to $\mathbb{Z} / 2 r(K)$ such that at each cusp, $m$ increases by 1 from the lower to the upper strand. Near a crossing $q$, let $\alpha$ and $\beta$ be the strands of $D$ with, respectively, more negative and more positive slope. Assign the grading $|q| \equiv m(\alpha)-m(\beta)(\bmod 2 r(K))$. For instance, all three crossings in the trefoil of Figure 1 have degree 0 . (The other generators of $\mathscr{A}$, the right cusps, are all assigned grading 1.) The central result of [Chekanov 2002a] states that $(\mathscr{A}, \partial)$ is invariant under Legendrian isotopy, up to stable tame isomorphism.

Henceforth we assume that we have an integer $\rho$ dividing $2 r(K)$. In this case, it makes sense to discuss whether a crossing of $D$ (a generator of $\mathscr{A}$ ) has degree divisible by $\rho$.

One way to extract information from $(\mathscr{A}, \partial)$ is via augmentations. A $\rho$-graded augmentation of $(\mathscr{A}, \partial)$ is an algebra map $\varepsilon: \mathscr{A} \rightarrow \mathbb{Z} / 2$ such that $\varepsilon \circ \partial=0, \varepsilon(1)=1$, and $\varepsilon(a)=0$ if $\rho \nmid|a|$. (This last condition states that $\varepsilon$ respects the grading of 
$\mathscr{A}$ over $\mathbb{Z} / \rho$, where the base ring $\mathbb{Z} / 2$ is considered to lie in degree 0 .) Two cases are of special interest. If $\rho=1$, then a 1-graded augmentation is also known as an ungraded augmentation, on which any generator can be augmented (i.e., sent to 1 by $\varepsilon$ ) regardless of grading. If $\rho=0$, and hence $r(K)=0$, then a 0 -graded augmentation is also known as a $\mathbb{Z}$-graded augmentation, on which only generators of degree $0 \in \mathbb{Z}$ can be augmented.

Note that, since $\mathscr{A}$ is finitely generated as an algebra, any DGA has only finitely many $\rho$-graded augmentations. The number of $\rho$-graded augmentations of $(\mathscr{A}, \partial)$ is invariant under tame isomorphism, and changes by a power of 2 under algebraic stabilization. More precisely, an algebraic stabilization of degree $i$ adds generators $\alpha, \beta$ of degree $i-1, i$ respectively, with $\partial(\beta)=\alpha$ and $\partial(\alpha)=0$. It follows that this stabilization leaves the number of $\rho$-graded augmentations unchanged if $\rho \nmid i$, and doubles this number if $\rho \mid i$.

If $\rho$ is odd or $\rho=0$, a normalized count of the number of $\rho$-graded augmentations gives us an invariant of $(\mathscr{A}, \partial)$ under stable tame isomorphism. Let $a_{k}$ be the number of generators of $\mathscr{A}$ of degree $k(\bmod \rho)$; the set $\left\{a_{k}\right\}_{k \in \mathbb{Z} / \rho}$ is called the degree distribution of the DGA. Set $\chi_{\rho}^{*}(\mathscr{A})$ to be the following "shifted Euler characteristic" of $\mathscr{A}$ :

and

$$
\chi_{0}^{*}(\mathscr{A})=\sum_{k \geq 0}(-1)^{k} a_{k}+\sum_{k<0}(-1)^{k+1} a_{k}
$$

$$
\chi_{\rho}^{*}(\mathscr{A})=\sum_{k=0}^{\rho-1}(-1)^{k} a_{k}
$$

if $\rho$ is odd. We can now define the following invariant of Legendrian isotopy:

Definition 1. Let $K$ be a Legendrian knot and $\rho$ a divisor of $2 r(K)$ with $\rho=0$ or $\rho$ odd. The normalized $\rho$-graded augmentation number of $K$, written $\operatorname{Aug}_{\rho}(K)$, is the product of $2^{-\chi_{\rho}^{*}(\mathscr{A}) / 2}$ and the number of $\rho$-graded augmentations of $(\mathscr{A}, \partial)$, where $(\mathscr{A}, \partial)$ is the contact homology DGA of $K$.

We remark that $\mathrm{Aug}_{0}$ distinguishes between Chekanov's well-known examples of Legendrian $5_{2}$ knots. Note that, when $\rho$ is even and nonzero, it is not possible to define similar normalized augmentation numbers to give an invariant under stable tame isomorphism. The issue in this case is that one can add an algebraic stabilization in each of degrees $0,2, \ldots, \rho-2$, or in each of degrees $1,3, \ldots, \rho-1$, and the degree distribution changes the same way in both cases; however, the number of augmentations doubles in the former case but remains the same in the latter.

We next turn to rulings. A ruling ${ }^{1}$ of a front is a decomposition of the front into pairs of paths beginning at a left cusp and ending at a right cusp that cobound disks.

\footnotetext{
${ }^{1}$ In [Chekanov 2002b] this is called an admissible decomposition and in [Pushkar and Chekanov 2005] it is called a positive proper decomposition.
} 
Each ruling path is smooth except at cusps and certain crossings called switches. Near a switch, the ruling disks must look like a diagram from the first row of Figure 2. If $\rho$ divides $2 r(K)$, the ruling is $\rho$-graded if all switches have degree divisible by $\rho$.

To each ruling $R$ of a front $D$ of a Legendrian knot $K$, we can associate an integer $\theta(R)=c(D)-s(R)$, where $c(D)$ is the number of right cusps of $D$ (i.e., the number of closed curves in $R$ ), and $s(R)$ is the number of switches of $R$. A main result of [Pushkar and Chekanov 2005] is that the multiset

$$
\Theta_{\rho}(K)=\{\theta(R) \mid R \text { is a } \rho \text {-graded ruling of } D\},
$$

which we call the complete ruling invariant of $K$, is invariant under Legendrian isotopy.

The complete ruling invariant is effective: Chekanov [2002b] used the complete ruling invariant to distinguish his original $5_{2}$ examples of Legendrian knots that have the same classical invariants. We conjecture in passing that when $\rho=1$ (i.e., the ungraded case), it seems possible that $\Theta_{1}(K)$ always depends only on the topological type and Thurston-Bennequin number of $K$. If this were true, then $\Theta_{1}$ would provide a topological knot invariant by considering any Legendrian $K$ in the knot type with maximal Thurston-Bennequin number. This is related to, but independent of, the conjecture in [ $\mathrm{Ng} 2003]$ that the ungraded abelianized characteristic algebra is a topological invariant, and the result of Rutherford [Rutherford 2005] that $\Theta_{1}(K)=\varnothing$ if and only if the Kauffman polynomial bound on the ThurstonBennequin number of $K$ is not sharp.

We are now in a position to state our main results.

Theorem 2. Given a Legendrian knot $K$ with a diagram $D$ in plat position, and an integer $\rho$ with $\rho \mid 2 r(K)$ and either $\rho=0$ or $\rho$ odd, there is a many-to-one correspondence between $\rho$-graded augmentations of the contact homology DGA $(\mathscr{A}, \partial)$ of $D$ and $\rho$-graded rulings of $D$, with $2^{\left(\theta(R)+\chi_{\rho}^{*}(\mathscr{A})\right) / 2}$ augmentations corresponding to a ruling $R$.

An example of the correspondence appears in Figure 1.

Theorem 2 has the following immediate consequence.

Corollary 3. Suppose $\rho \mid 2 r(K)$ and $\rho$ is zero or odd. The normalized $\rho$-graded augmentation number of a Legendrian knot $K$ can be deduced from the complete ruling invariant:

$$
\operatorname{Aug}_{\rho}(K)=\sum_{\theta \in \Theta_{\rho}(K)} 2^{\theta / 2}
$$

As stated earlier, it is possible that the complete ruling invariant is actually contained somehow in the contact homology DGA. One could hope to associate to each augmentation a fractional power of 2 , so that if a ruling gives $2^{k}$ augmentations 

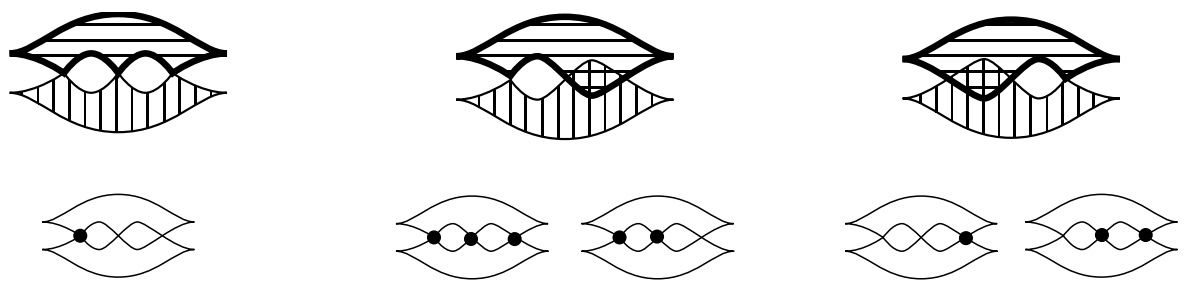

Figure 1. Many-to-one correspondence between $\mathbb{Z}$-graded augmentations and rulings on a Legendrian trefoil knot. Large dots represent augmented crossings. The trefoil shown has $r(K)=0$ and $\chi_{0}^{*}(\mathscr{A})=1$; the leftmost ruling has $\theta=-1$, the others $\theta=1$.

then each of those augmentations is given the fraction $2^{-k}$. This would allow us to recover the complete ruling invariant from the augmentations of the DGA.

\section{Proofs}

The proof of Theorem 2 rests on an algorithm described in [Sabloff 2005] that produces a ruling from an augmentation. After setting notation, we will describe this algorithm, use it to define the many-to-one correspondence in the theorem, and finally count the number of augmentations that correspond to each ruling in terms of the degree distribution of the contact homology DGA.

Let $K$ be a Legendrian knot with plat diagram $D$ and a $\rho$-graded ruling $R$. Near a crossing, call the two ruling paths that are incident to the crossing crossing paths and call the ruling paths that are paired with the crossing paths companion paths. At a crossing of $D$ whose grading is not divisible by $\rho$, the ruling simply passes through the crossing with no switch. We can use the ruling $R$ to partition the crossings of $D$ with grading divisible by $\rho$ into three types:

Switches: The ruling disks are nested or disjoint on both sides of the crossing. See the first line of Figure 2.

Departures: Moving left-to-right, the ruling disks pass from nested or disjoint to interlaced. See the second line of Figure 2.

Returns: Moving left-to-right, the ruling disks pass from interlaced to nested or disjoint. See the third line of Figure 2.

Henceforth the terms "switches", "departures", and "returns" will refer to crossings of grading divisible by $\rho$ with the above properties.

The correspondence in Theorem 2 is derived from the procedure implicitly described in [Sabloff 2005, §3.3, especially the remark at the end of the section] for producing a ruling on a plat diagram from an augmentation $\varepsilon$. We recall the 


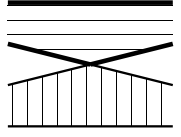

(S1)

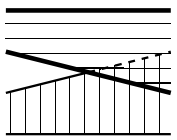

(D1)

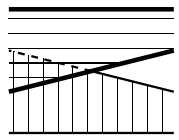

(R1)

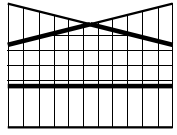

(S2)



(D2)

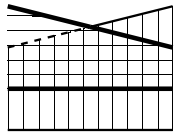

(R2)



(S3)

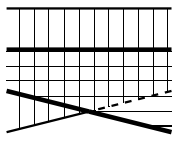

(D3)

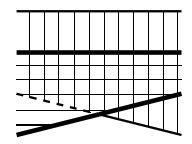

(R3)

Figure 2. Switches (top), departures (middle), and returns (bottom).

procedure here. Label the crossings from left to right by $q_{1}, \ldots, q_{n}$. The construction begins at the left cusps, where any ruling must pair paths incident to the same cusp. The next step is to extend the ruling over the crossings from left to right, one crossing at a time. The extension procedure uses a succession of "virtual augmentations", $\rho$-graded algebra maps $\varepsilon_{j}: \mathscr{A} \rightarrow \mathbb{Z} / 2$, where $\varepsilon_{1}=\varepsilon$ and $\varepsilon_{j+1}$ is defined after the ruling has been extended over $q_{j}$.

The extension of the ruling over $q_{j}$ depends on the configuration of the ruling disks to the left of $q_{j}$ and on $\varepsilon_{j}\left(q_{j}\right)$. If $\left|q_{j}\right|$ is not divisible by $\rho$, then the ruling extends without switching. Otherwise, the ruling extends according to the following instructions:

- If the ruling disks are nested or disjoint to the left of $q_{j}$ and $\varepsilon_{j}\left(q_{j}\right)=1$, extend the ruling over the crossing as a switch.

- If the ruling disks are nested or disjoint to the left of $q_{j}$ and $\varepsilon_{j}\left(q_{j}\right)=0$, extend the ruling over the crossing as a departure.

- Otherwise - i.e., if the ruling disks are interlaced to the left of $q_{j}$ - extend the ruling over the crossing as a return.

Once the ruling has been extended over $q_{j}$, we define the virtual augmentation $\varepsilon_{j+1}$. If $\varepsilon_{j}\left(q_{j}\right)=0$, or if the ruling is extended over $q_{j}$ as in configuration (R1), then set $\varepsilon_{j+1}=\varepsilon_{j}$. For the other configurations, set $\varepsilon_{j+1}\left(q_{k}\right)=\varepsilon_{j}\left(q_{k}\right)$ for $k \leq j$; we now inductively define $\varepsilon_{j+1}\left(q_{k}\right)$ for $k \geq j+1$.

Suppose that $\varepsilon_{j+1}\left(q_{l}\right)$ has been defined for $l<k$. The value of $\varepsilon_{j+1}\left(q_{k}\right)$ depends on a count of a special set of embeddings of the 2-disk into the diagram, up to smooth reparametrization. If the number of these disks is odd, $\varepsilon_{j+1}\left(q_{k}\right)$ 


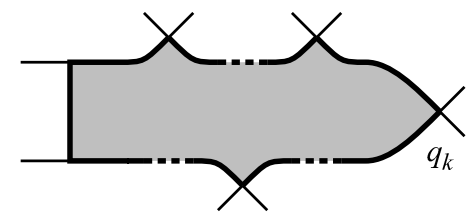

Figure 3. The embedding of the disk used to determine the new virtual augmentation $\varepsilon_{j+1}$ on $q_{k}$. The vertical segment lies to the right of $q_{j}$ and joins either the crossing strands or the companion strands; in the latter case, some other strands may cross the vertical segment. The corners on the top and bottom lie at crossings where $\varepsilon_{j+1}=1$.

equals $\varepsilon_{j}\left(q_{k}\right)+1$; the two virtual augmentations agree on $q_{k}$ otherwise. The image of the boundary of the disks leaves $q_{k}$ on the upper left strand, travels leftwards (possibly with convex corners at vertices with $\varepsilon_{j+1}=1$ ) to the top of a vertical line segment that joins two strands of the diagram and lies just to the right of $q_{j}$. The boundary then traverses this segment and travels rightwards (again with possible convex corners where $\varepsilon_{j+1}=1$ ) back to $q_{k}$. See Figure 3 . In the case where the ruling has been extended over $q_{j}$ in configurations (R2) or (R3), the vertical segment above must join the companion strands. In the case of configuration (S1), the vertical segment joins the crossing strands. In the case of configuration (S2), first perform the entire inductive procedure using disks with vertical segments that join the companion strands, to obtain a virtual augmentation $\varepsilon_{j+1}^{\prime}$ from $\varepsilon_{j}$; then repeat the procedure with vertical segments that join the crossing strands, to obtain the virtual augmentation $\varepsilon_{j+1}$ from $\varepsilon_{j+1}^{\prime}$. For configuration (S3), do the same thing in the opposite order. It was proven in [Sabloff 2005] that this procedure determines a genuine ruling.

With the algorithm now in hand, we may begin the proof of the correspondence between augmentations and rulings. The algorithm sends an augmentation to a ruling; the question is which augmentations get sent to the same ruling. The answer is given by the following:

Lemma 4. If a $\rho$-graded ruling of a plat diagram $D$ has $r$ returns, then the number of $\rho$-graded augmentations corresponding to it is $2^{r}$ if $\rho \neq 1$, and $2^{r+c(D)}$ if $\rho=1$.

Proof. The algorithm given above for obtaining a ruling $R$ from an augmentation $\varepsilon$ also gives a "final" virtual augmentation $\varepsilon_{n}$. Different augmentations result in different final virtual augmentations; simply consider the leftmost crossing $q_{k}$ at which the augmentations differ. It is also clear from the algorithm that $\varepsilon_{n}$ augments the switches of $R$, some subset of the returns of $R$, and no other crossings. 
We claim that any virtual augmentation which augments only the switches and some subset of the returns of $R$, for some ruling $R$, is the final virtual augmentation for some augmentation of the diagram. Indeed, the argument in [Sabloff 2005] leading to the algorithm shows that such a virtual augmentation $\varepsilon^{\prime}$ corresponds to an honest augmentation on the DGA $\left(\mathscr{A}^{\prime}, \partial^{\prime}\right)$ for a different ("dipped") diagram for the knot. The algorithm determines a stable tame isomorphism between the DGA $(\mathscr{A}, \partial)$ for $D$ and $\left(\mathscr{A}^{\prime}, \partial^{\prime}\right)$. Thus, the augmentation on $\left(\mathscr{A}^{\prime}, \partial^{\prime}\right)$ yields an augmentation $\varepsilon$ on a stabilization of $(\mathscr{A}, \partial)$ and hence, by restriction, on $(\mathscr{A}, \partial)$ itself. Then $\varepsilon^{\prime}$ is the final virtual augmentation for $\varepsilon$, proving the claim.

Now given a $\rho$-graded ruling $R$, the number of virtual augmentations augmenting only its switches and some of its returns is clearly $2^{r}$ when $\rho \neq 1$, and $2^{r+c(D)}$ when $\rho=1$, since in the latter case any subset of the right cusps, which have degree 1, can be augmented. By the preceding argument, this is also the number of augmentations corresponding to $R$.

Theorem 2 now follows from Lemma 4 and the following combinatorial result.

Lemma 5. The number of returns of a $\rho$-graded ruling $R$ is $\frac{1}{2}\left(\theta(R)+\chi_{\rho}^{*}(A)\right)$ if $\rho=0$ or $\rho \geq 3$ is odd, and $\frac{1}{2}\left(\theta(R)+\chi_{1}^{*}(A)\right)-c(D)$ if $\rho=1$.

Proof. As before, let $r$ be the number of returns of a $\rho$-graded ruling $R$; also, let $s$ and $d$ denote the number of switches and departures, respectively (by definition, this only counts crossings of degree divisible by $\rho$ ).

Consider a vertical line which intersects the plat diagram generically (i.e., it does not pass through a crossing or cusp). Since there are $c(D)$ right cusps, any such line intersects the diagram in $2 c(D)$ points, ordered from top to bottom, and the ruling determines a pairing of these points. In addition, each point $p$ carries a Maslov index $m(p)$ given by the Maslov index of its strand (see [Chekanov 2002b] or [Sabloff 2005] for the definition of Maslov index), and the Maslov indices of paired points differ by 1 . Say that two pairs of points are interlaced if we encounter the pairs alternately as we move from top to bottom; that is, they appear from top to bottom as $a_{1} b_{1} a_{2} b_{2}$, where $a_{i}$ denotes one pair of companion strands and $b_{i}$ denotes the other. To any generic vertical line we associate a number called the interlacing number. We will see that as we move the vertical line from left to right, beginning just after the left cusps and ending just before the right cusps, then the interlacing number begins and ends at 0 , and changes by \pm 1 when passing through each crossing; counting these changes yields the lemma.

We first consider the case $\rho=1$, i.e., the ungraded case. Here we define the interlacing number of a vertical line to be the number of interlaced pairs on it. As we sweep the vertical line from left to right, the interlacing number clearly begins and ends at 0 , is unchanged at each switch, increases by 1 at each departure, and 
decreases by 1 at each return. Thus $d=r$; the lemma follows from the fact that $\chi_{1}^{*}(A)=a_{0}=s+d+r+c(D)$.

Next consider the case $\rho=0$, i.e., the $\mathbb{Z}$-graded case. For $k \in \mathbb{Z}$, let $\tilde{a}_{k}$ be the number of crossings of $D$ of degree $k$, and note that, for $a_{k}$ defined as in Section 1, we have $\tilde{a}_{k}=a_{k}$ for $k \neq 1$ and $a_{1}-\tilde{a}_{1}=c(D)$; also, $a_{0}=s+d+r$ and $\theta(R)=c(D)-s$. The lemma now reduces to proving that

$$
d-r+\sum_{k>0}(-1)^{k} \tilde{a}_{k}+\sum_{k<0}(-1)^{k+1} \tilde{a}_{k}=0 .
$$

To two interlaced pairs $a_{1} b_{1} a_{2} b_{2}$ we associate a sign:

$$
\operatorname{sgn}\left(a_{1} b_{1} a_{2} b_{2}\right)=\left\{\begin{aligned}
1 & \text { if } m\left(a_{2}\right)-m\left(b_{2}\right) \in\{\ldots,-4,-2,0,1,3,5, \ldots\}, \\
-1 & \text { if } m\left(a_{2}\right)-m\left(b_{2}\right) \in\{\ldots,-5,-3,-1,2,4,6, \ldots\} .
\end{aligned}\right.
$$

Define the interlacing number of a vertical line to be the sum over all interlaced pairs of this sign. Again, if we move the vertical line from left to right, the interlacing number begins and ends at 0 , and changes only when the line passes through a nonswitch crossing.

Consider any crossing $C$ besides a switch. We claim that the interlacing number changes at $C$ by \pm 1 , and that each crossing contributes the appropriate sign to obtain $(*)$. If we ignore degree, $C$ looks like one of $\left(\mathrm{D}^{*}\right)$ or $\left(\mathrm{R}^{*}\right)$ from Figure 2. There is a one-to-one correspondence between interlaced pairs as for switches, except for one extra interlaced pair on the right of $C$, in the case of $\left(D^{*}\right)$, or on the left, in the case of ( $\left.\mathrm{R}^{*}\right)$. Label this interlaced pair by $a_{1} b_{1} a_{2} b_{2}$. As we pass through $C$, the interlacing number changes by $\operatorname{sgn}\left(a_{1} b_{1} a_{2} b_{2}\right)$ for $\left(\mathrm{D}^{*}\right)$ and $-\operatorname{sgn}\left(a_{1} b_{1} a_{2} b_{2}\right)$ for $\left(\mathrm{R}^{*}\right)$.

Recall from Section 2 that the degree $|\cdot|$ of a crossing is the difference of the Maslov indices of the crossing strands, more precisely $m(a)-m(b)$ where $a$ lies above $b$ to the left of the crossing. Note also that for the interlaced pair $a_{1} b_{1} a_{2} b_{2}$ as above, we have $m\left(a_{1}\right)=m\left(a_{2}\right)+1$ and $m\left(b_{1}\right)=m\left(b_{2}\right)+1$. It follows that the degree of a crossing that looks like (D1) is $m\left(a_{2}\right)-m\left(b_{2}\right)-1$; (D2) and (D3), $m\left(b_{2}\right)-m\left(a_{2}\right)$; (R1), $m\left(b_{2}\right)-m\left(a_{2}\right)+1 ;(\mathrm{R} 2)$ and (R3), $m\left(a_{2}\right)-m\left(b_{2}\right)$. An easy computation using the definition of $\operatorname{sgn}\left(a_{1} b_{1} a_{2} b_{2}\right)$ shows that the interlacing number changes by:

- +1 if $|C| \in\{\ldots,-5,-3,-1,2,4,6, \ldots\}$, or $|C|=0$ and $C$ is a departure,

- -1 if $|C| \in\{\ldots,-6,-4,-2,1,3,5, \ldots\}$, or $|C|=0$ and $C$ is a return.

Since the interlacing begins on the left as 0 and ends on the right as 0 , counting each of these changes of \pm 1 yields $(*)$, as desired. This completes the proof when $\rho=0$. 
The proof of the lemma when $\rho \geq 3$ is odd is identical to the proof when $\rho=0$, except that we define

$$
\operatorname{sgn}\left(a_{1} b_{1} a_{2} b_{2}\right)=\left\{\begin{aligned}
1 & \text { if } m\left(a_{2}\right)-m\left(b_{2}\right) \in\{0,1,3,5, \ldots, \rho-2\} \\
-1 & \text { if } m\left(a_{2}\right)-m\left(b_{2}\right) \in\{2,4,6, \ldots, \rho-1\}
\end{aligned}\right.
$$

\section{References}

[Chekanov 2002a] Y. Chekanov, "Differential algebra of Legendrian links", Invent. Math. 150:3 (2002), 441-483. MR 2003m:53153 Zbl 1029.57011

[Chekanov 2002b] Y. V. Chekanov, "Invariants of Legendrian knots", pp. 385-394 in Proceedings of the International Congress of Mathematicians (Beijing, 2002), vol. II, edited by T. Li, Higher Ed. Press, Beijing, 2002. MR 2004a:57015 Zbl 0998.57046

[Eliashberg 1998] Y. Eliashberg, "Invariants in contact topology", Doc. Math. Extra Vol. II (1998), 327-338. MR 2000a:57068 Zbl 0913.53010

[Fuchs 2003] D. Fuchs, "Chekanov-Eliashberg invariant of Legendrian knots: existence of augmentations”, J. Geom. Phys. 47:1 (2003), 43-65. MR 2004h:57007 Zbl 1028.57005

[Fuchs and Ishkhanov 2004] D. Fuchs and T. Ishkhanov, "Invariants of Legendrian knots and decompositions of front diagrams”, Mosc. Math. J. 4 (2004), 707-717. MR 2005i:57033 Zbl 1073.53106

[Ng 2003] L. L. Ng, “Computable Legendrian invariants", Topology 42:1 (2003), 55-82. MR 2003h: 57038 Zbl 1032.53070

[Ng and Traynor 2004] L. Ng and L. Traynor, "Legendrian solid-torus links", J. Symplectic Geom. 2:3 (2004), 411-443. MR 2005k:57051 Zbl 02199036

[Pushkar and Chekanov 2005] P. E. Pushkar' and Y. V. Chekanov, "Combinatorics of fronts of Legendrian links, and the Arnol'd 4-conjectures”, Uspekhi Mat. Nauk 60:1 (2005), 99-154. In Russian; translated in Russian Math. Surveys 60:1 (2005), 95-149. MR 2145660 Zbl 02194118

[Rutherford 2005] D. Rutherford, “The Bennequin number, Kauffman polynomial, and ruling invariants of a Legendrian link: the Fuchs conjecture and beyond", preprint, 2005. math.GT/0511097

[Sabloff 2005] J. M. Sabloff, "Augmentations and rulings of Legendrian knots", Int. Math. Res. Not. 2005:19 (2005), 1157-1180. MR 2147057 Zbl 02188817

Received March 9, 2005. Revised July 29, 2005.

LENHARD L. NG

DEPARTMENT OF MATHEMATICS

STANFORD UNIVERSITY

450 SerRa MALL, BLDG. 380

STANFORD, CA 94305

UNITED STATES

lng@math.stanford.edu

JOSHUA M. SABLOFF

DEPARTMENT OF MATHEMATICS

HAVERFORD COLLEGE

370 Lancaster Ave.

HAVERFORD, PA 19041

UNITED STATES

jsabloff@haverford.edu 